\title{
Role of the Microbiome in Celiac Disease
}

\author{
Nóra Judit Béres ${ }^{1}$, Erna Sziksz ${ }^{1,2}$, Ádám Vannay ${ }^{1,2}$, Dolóresz Szabó ${ }^{1}$, Domonkos Pap ${ }^{1,2}$, Apor Veres-Székely ${ }^{1,2}$, \\ András Arató ${ }^{1}$, Attila J. Szabó ${ }^{1,2}$, Gábor Veres ${ }^{1, *}$
}

\author{
(Nóra Judit Béres ${ }^{1}$, Erna Sziksz ${ }^{1,2}$ - equally contributed first authors) \\ ${ }^{1}$ Department of Pediatrics, Semmelweis University, H-1083, Budapest, Hungary \\ ${ }^{2}$ MTA-SE,Pediatrics and Nephrology Research Group, H-1083, Budapest, Hungary \\ *Corresponding author: veres.gabor@med.semmelweis-univ.hu
}

Received October 10, 2014; Revised October 16, 2014; Accepted October 17, 2014

\begin{abstract}
Microbiome is the community of commensal, symbiotic and pathogenic microorganism that share our human body space. Intestinal microbiota has a defensive role in human health, it is implicated in metabolic and nutritional processes and plays an important role in the pathophysiology of several diseases. In recent years special attention has been paid to investigations targeting the changes of intestinal microbiome in various gastrointestinal disorders including inflammatory bowel disease, infectious colitis and celiac disease (CD). The aim of our present review is to summarize the role of the microbiome in $\mathrm{CD}$ and the changes of its composition in the intestine of patients suffering from CD.
\end{abstract}

Keywords: microbiome, intestinal microbiota, celiac disease

Cite This Article: Nóra Judit Béres, Erna Sziksz, Ádám Vannay, Dolóresz Szabó, Domonkos Pap, Apor Veres-Székely, András Arató, Attila J. Szabó, and Gábor Veres, "Role of the Microbiome in Celiac Disease." International Journal of Celiac Disease, vol. 2, no. 4 (2014): 150-153. doi: 10.12691/ijcd-2-4-4.

\section{Introduction}

Celiac disease (CD) also known as gluten-sensitive enteropathy or nontropical sprue is a chronic immunemediated enteropathy of the small intestine induced by dietary gluten and related prolamins [1,2]. Most of the prolamin proteins - present in wheat, rye and barley - may contain immunogenic epitopes causing adverse symptoms of CD through the induction of inflammatory responses of the intestinal mucosa which lead to villous atrophy and malabsorption $[3,4,5,6]$.

CD develops more frequently in genetically predisposed individuals, it has been associated with the major histocompatibility complex region. More than $90 \%$ of $\mathrm{CD}$ patients express the antigen-presenting molecules human leukocyte antigen (HLA)-DQ-2/2.2/8, however only in a part of HLA-DQ-2/2.2/8 positive individuals develops CD $(40 \%$ of the population carries these haplotypes without any evidence of CD) [6]. Based on recent epidemiological studies, similarly to other immunemediated diseases (allergy or asthma) CD may appear at any age and its prevalence is continuously increasing [1]. These observations assume that beside genetic predisposition there are other factors which contribute to the pathogenesis of CD such as environmental factors, diet and microbiome [5]. The aim of the recent review is to introduce the possible role of the microbiome in the gut and in the pathomechanism of CD.

\section{Microbiome in the Gut}

The human body is inhabited by a large amount of bacteria, viruses and archaea. Most of them are localized in the gastrointestinal tract (colon contains $70 \%$ of all microbes). Gut colonization starts during birth with the passage through the birth canal and is followed by a gradual microbiome change until young adulthood. Caesarian delivery can also affect the early biodiversity of the intestine [7]. The composition of the microbiome depends on genetic background, geographic determinants, and diet as well $[8,9]$.

Gut is the primary site of the interaction between the microorganisms and host immune system [10] and the balanced intestinal microbiome is responsible for the physiological development of the (gut) immune system. The intestinal microbiome promotes immune maturation and tolerance through the activation of immune responses leading to the expression of immunoglobulins and cytokines including interleukin-8, -10, -17, -21, -22, and interferon- $\gamma[5,11]$.

The luminal surface of the gut is covered by a mucous layer, which contains binding sites for commensal microbes and prevents the direct contact of the enterocytes with pathogens [8]. Moreover, bacteria and their components and metabolic products - defensins, cathedicilins, shortchain fatty acids - provide the host to stimulate the production of antimicrobial substances [8]. Examination of faecal samples from healthy individuals revealed 
limited overlap in the microbial composition between the individuals, but certain phylotypes occur more commonly than others. The most common bacteria in the healthy duodenum are the Bacilli, Streptococcaceae, Actinobacteria, Actinomycinaeae and Corynebacteriaceae in bacterial concentration of $10^{3}$ cells/g [8].

Identification of the dominant intestinal bacterial species and the effect of diet, age, body weight and drugs (antibiotics, steroids) on the colonization are crucial to uncover the impact of the gut microbiota on human health $[12,13]$. Clinical and experimental studies confirmed that the gut microbiota plays an important role also in the pathogenesis of several disorders including obesity, diabetes, atopic diseases and it contributes to the development of CD as well $[11,14]$.

The bacterial peptide glycans and polysaccharides are recognized by antigen presenting cells through their specific pattern recognition receptors including toll-like receptors (TLRs) and their co-receptor the cluster of differentiation 14 (CD14). Activation of these receptors contributes to the increased expression of inflammatory cytokines, initiating infiltration of lymphocytes into the lamina propria, villous atrophy, crypt hyperplasia, which are all characteristic for CD [15]. Also toxic gliadin fragments may induce TLR signaling and regulates the expression of tight junction proteins. The activation of those pathways can lead to the damage of the epithelial barrier and increased intestinal permeability, which facilitate the access of higher antigen (bacterial antigens and prolamins) loads [5].

\section{Detection of the Intestinal Microbiome}

Examination of the intestinal microbiota is complicated due to the dominance of anaerob bacteria and the difficulties of their culturing [16]. In the past few years the revolution of gene sequencing and bacterial phylogenetic microarrays made it possible to comprehensively study the human intestinal microbiome through the identification of bacteria-specific regions with oligonucleotide probes [17]. After DNA extracting and next-generation sequencing of the bacterial $16 \mathrm{~S}$ ribosomal (r)RNA more accurate and realistic results compared to regular bacterial cultures can be obtained [16]. 16S rRNA, a component of prokaryotic ribosomes is used as the most common genetic marker, which contains nine "hyper-variable" regions and speciesspecific sequences within a variable region. These speciesspecific sequences serve astargets for scientific research and diagnostic assay developmentallowing us the comprehensive and high revolution analysis of microbiota composition $[5,18]$.

\section{Microbiome in CD}

Recent studies demonstrated that the composition of the intestinal microbiota in patients with CD differs from that of healthy individuals [5]. The gastrointestinal tract is covered by a mucous glycocalyx layer, which carbohydrate structures represents attachment sites for host bacteria [3]. Inherited differences in the expression of carbohydrates may lead to the colonization of certain strains of bacteria, which influences the susceptibility of the individuals to
CD. Also the presence of certain bacterium species may cause environmental changes predisposing CD independently from genetic background through the alteration of carbohydrate expression [3]. Indeed the ratio of beneficial to harmful bacterial strains was significantly lower in patients with CD compared to healthy controls and was intermediate in patients following gluten free diet (GFD) [19].

De Palma et al. measured the effect of GFD over a month in healthy subjects, they found out that GFD led to the reduction of beneficial gut bacteria populations, which is in contrary to the results in CD patients. They also found out that GFD stimulates the host immunity [20].

Differences in the microbiota of patients with CD compared to healthy controls have been studied mostly in faeces, however there is an increased number of studies in the local, mucosa associated bacterial composition from biopsies. Recent studies investigating the major bacterial phylotypes in the duodenal mucosa of CD patients compared to healthy individuals demonstrated that Proteobacteria, Bacilli and Bacteroidetes were dominant in both groups and there was no significant difference between the bacterial diversity of each groups in major phylotypes [5]. Investigating the sub-population profile they found eight genus-like bacterial groups in duodenal biopsies which distinguish CD patients from healthy controls. Bacteria in the duodenum of patients with CD are Prevotella, Haemphilus, Serratia, that of healthy controls are Prevotellaoralis et rel., Proteus et rel., Clostridium stercorarium et rel., Ruminococcusbromii et rel., Papillibactercinnamivorans et rel. [5].

Patients with active CD had a higher amount of total bacteria, increased level of harmful Gram-negative bacteria (Bacteroides, Prevotella, Escherichia (E.) coli) and a lower number of Gram-positive bacteria including the protective Lactobacilli and Bifidobacteria [8,19,21,22]. This dysbiosis was only partially normalized after GFD [23].

Similarly, Nadal et al. and Collado et al. studying the intestinal microbiome found also that Bacterioidetes and $E$. coliwere significantly more abundant in the duodenal biopsies from pediatric patients with CD, which returned to normal in patients who had been on GFD for 1-2 years [19]. Also Collado et al. found that the local and faecal microbiota composition were similar [21]. Increased level of Bacteroides and Clostridium without any increment in the amount of $E$. coli was also detected in biopsies and faeces in GFD-treated patients compared to the CD group. $E$. coli maybe associated with the active phase of the disease generating mucosal lesions and atrophy [21]. Recent studies confirmed that the level of rod-shaped bacteria (Bacillus, Firmicutes) was elevated in the duodenal mucosa of pediatric patients with active CD and in GFD-treated children as well [24].

Sanchez et al. confirmed that there is a lower level of the protective phylum Firmicutes and Streptococceae and an elevated level of Proteobacteria, Enterobacteriacae, Staphylococceae and Klebsiella in patients with CD [25]. The same research group examined the differences in Bacteroides spp. and their pathogenic features confirm an increased abundance of Bacterioides fragilis strains with metalloprotease activity [22,25]. They demonstrated that patients with active CD showed the highest Renyi diversity values followed by the non-active CD patients 
and healthy individuals, which was used to determine differences in bacterium species among study groups. Renyi diversity index was lower in the duodenal biopsies of patients maintained on GFD and control individuals [25].

In conclusion, there are significant differences between the duodenal bacterial composition of patients with newly diagnosed CD and that of treated patients maintained on GFD. Lifelong lasting GFD, which is recently the only effective treatment of $\mathrm{CD}$, only partially contribute to the restoration of the intestinal microbiome, the imbalance is maintained even after treatment [22].

The prevalence of small intestine bacterial overgrowth (SIBO) in CD is not well known, because of the limitations in diagnosis, but it is considered as a potential cause for non-responsive CD [26]. Galatola et al. have shown a high prevalence of SIBO in CD (25\%), and another studies demonstrated even higher prevalence in 15 non-responsive CD (66\%) [27,28]. Following antibiotic (rifaximin) treatment the patients with non-responsive CD were symptom free [28].

The microbiota of infants, which were genetically predisposed (HLA-DQ-2 and/or 8) to CD, differs from that of non-predisposed children. Recent studies reported a reduced amount of Bacteroides, Escherichi/Shigella and Clostridiumin genetically predisposed infants [29]. In contrary another study showed increased level of Bacteroides fragilis and Staphylococci and decreased amount of Bifidobacteria in genetically susceptible infants.

Palma et al. also found a difference between the microbiome compositions of breast-fed (Bifidobacterium species) or formula-fed (Enterobacteriaceae, Streptococcus, Bacteroides, Clostridium, and Bifidobacterium) infants with and without carrying HLA-DQ-2 haplotypes. Moreover breast-feeding reduced the genotype-related differences in microbiota composition which may partly explain the protective role of breast milk in many disorders [30]. These studies suggest that the genotype and diet determine the gut colonisers. Main changes in the microbiome composition found in patients with CD compared to controls are shown in Table 1.

Table 1. Microbiome changes in CD. The table shows major groups of microbes that were increased or decreased in patients with active CD compared to control individuals

\begin{tabular}{|l|l|}
\hline Increased in CD & Decreased in CD \\
\hline Bacteroides [8,19,21] & Lactobacilli [8,19,21] \\
\hline Proteobacteria [25] & Bifidobacteria [8,19,21] \\
\hline Prevotella [5] & Streptococcaceae [25] \\
\hline Haemphilus [5] & Firmicutes [25] \\
\hline Serratia [5] & \\
\hline E. Coli [19] & \\
\hline Enterobacteriacae [25] & \\
\hline Stapyholoccaceae [25] & \\
\hline Klebsiella [25] & \\
\hline Bacilli [5] & \\
\hline Bacterioidetes [5] & \\
\hline Clostridium [21] & \\
\hline
\end{tabular}

Further studies are required to explore the complex functions of the microbiome, there cognition of the bacterial dysbiosis in patients with CD led to the exploration of possible benefits of probiotic formulas to improve the symptoms of CD.

\section{Conclusions}

Overall it seems to be unequivocal, that $\mathrm{CD}$ is associated with the overgrowth of pathobionts resulting in decreased number of protective symbiont and commensal colonies in the small intestine. Alteration of the intestinal microbiota may be consequence of the massive destruction of the duodenal mucosa, the release of proinflammatory markers and other cell-derived factors, which may foster the colonization of harmful Gram-negative bacteria in genetically predisposed individuals inducing the loss of tolerance against prolamins.

The severity of the disease also determines the composition of the microbiome parallel to the activation or remission of the mucosal lesions suggesting that the presence of some bacterium species may be a biological marker of disease activity.

\section{Acknowledgement}

This work was supported by Hungarian National Scientific Research Foundation Grant - OTKA K108688, K100909, K105530, K108655, PD105361, KMR_12-12012-0074 and LP008/2014.

\section{Statement of Competing Interests}

The authors declare no competing interests.

\section{References}

[1] Rosen A, Sandstrom O, Carlsson A, Hogberg L, Olen O, Stenlund $\mathrm{H}$, et al. Usefulness of Symptoms to Screen for Celiac Disease. Pediatrics 2014; 133: 211-218.

[2] Sziksz E., Vörös P., Veres G., Fekete A., Vannay Á. Coeliac Disease: From Triggering Factors to Treatment. International Journal of Celiac Disease 2013; 1: 9-13.

[3] Toft-Hansen H, Nielsen C, Biagini M, Husby S, Lillevang ST. Lectin staining shows no evidence of involvement of glycocalyx/mucous layer carbohydrate structures in development of celiac disease. Nutrients 2013; 5: 4540-4552.

[4] Kocsis D, Miheller P, Lorinczy K, Herszenyi L, Tulassay Z, Racz $\mathrm{K}$, et al. Coeliac disease in a 15-year period of observation (1997 and 2011) in a Hungarian referral centre. European journal of internal medicine 2013; 24: 461-467.

[5] Cheng J, Kalliomaki M, Heilig HG, Palva A, Lahteenoja H, de Vos WM, et al. Duodenal microbiota composition and mucosal homeostasis in pediatric celiac disease. BMC gastroenterology 2013; 13: 113.

[6] Kocsis D, Beres N, Veres G, Szabo D, Muller KE, Arato A, et al. [Genetic and epigenetic aspects of celiac disease]. Orv Hetil 2014; 155: 83-88.

[7] Biasucci G, Benenati B, Morelli L, Bessi E, Boehm G. Cesarean delivery may affect the early biodiversity of intestinal bacteria. The Journal of nutrition 2008; 138: 1796s-800s.

[8] Bustos Fernandez LM, Lasa JS, Man F. Intestinal Microbiota: Its Role in Digestive Diseases. Journal of clinical gastroenterology 2014; 48: 657-666.

[9] Di Gioia D, Aloisio I, Mazzola G, Biavati B. Bifidobacteria: their impact on gut microbiota composition and their applications as probiotics in infants. Applied microbiology and biotechnology. 2014; 98: 563-577.

[10] Round JL, Mazmanian SK. The gut microbiota shapes intestinal immune responses during health and disease. Nature reviews Immunology 2009; 9: 313-323.

[11] Vieira AT, Teixeira MM, Martins FS. The Role of Probiotics and Prebiotics in Inducing Gut Immunity. Frontiers in immunology 2013; 4: 445. 
[12] Flint HJ, Duncan $\mathrm{SH}$, Scott KP, Louis P. Interactions and competition within the microbial community of the human colon: links between diet and health. Environmental microbiology 2007; 9: 1101-1111.

[13] Sokol H, Pigneur B, Watterlot L, Lakhdari O, Bermudez-Humaran LG, Gratadoux JJ, et al. Faecalibacterium prausnitzii is an antiinflammatory commensal bacterium identified by gut microbiota analysis of Crohn disease patients. Proceedings of the National Academy of Sciences of the United States of America 2008; 105: 16731-16736.

[14] Rajpal DK, Brown JR. Modulating the human gut microbiome as an emerging therapeutic paradigm. Science progress 2013; 96: 224-236.

[15] Dezsofi A, Szebeni B, Hermann CS, Kapitany A, Veres G, Sipka $\mathrm{S}$, et al. Frequencies of genetic polymorphisms of TLR4 and CD14 and of HLA-DQ genotypes in children with celiac disease, type 1 diabetes mellitus, or both. Journal of pediatric gastroenterology and nutrition 2008; 47: 283-287.

[16] Goodman AL, Kallstrom G, Faith JJ, Reyes A, Moore A, Dantas $\mathrm{G}$, et al. Extensive personal human gut microbiota culture collections characterized and manipulated in gnotobiotic mice. Proceedings of the National Academy of Sciences of the United States of America 2011; 108: 6252-6257.

[17] Major G, Spiller R. Irritable bowel syndrome, inflammatory bowel disease and the microbiome. Current opinion in endocrinology, diabetes, and obesity 2014; $21: 15-21$

[18] Chakravorty S, Helb D, Burday M, Connell N, Alland D. A detailed analysis of $16 \mathrm{~S}$ ribosomal RNA gene segments for the diagnosis of pathogenic bacteria. Journal of microbiological methods 2007; 69: 330-339.

[19] Nadal I, Donat E, Ribes-Koninckx C, Calabuig M, Sanz Y. Imbalance in the composition of the duodenal microbiota of children with coeliac disease. Journal of medical microbiology. 2007 56: 1669-74.

[20] De Palma G, Nadal I, Collado MC, Sanz Y. Effects of a glutenfree diet on gut microbiota and immune function in healthy adult human subjects. The British journal of nutrition 2009; 102: 11541160.

[21] Collado MC, Donat E, Ribes-Koninckx C, Calabuig M, Sanz Y. Specific duodenal and faecal bacterial groups associated with paediatric coeliac disease. Journal of clinical pathology 2009; 62: 264-269.

[22] Lupan I, Sur G, Deleanu D, Cristea V, Samasca G, Makovicky P. et al. Celiac disease microbiota and its applications. Ann Microbiol 2013; 64: 899-903.

[23] Olivares M, Castillejo G, Varea V, Sanz Y. Double-blind, randomised, placebo-controlled intervention trial to evaluate the effects of Bifidobacterium longum CECT 7347 in children with newly diagnosed coeliac disease. The British journal of nutrition 2014; 112: 30-40.

[24] Forsberg G, Fahlgren A, Horstedt P, Hammarstrom S, Hernell O, Hammarstrom ML. Presence of bacteria and innate immunity of intestinal epithelium in childhood celiac disease. The American journal of gastroenterology 2004; 99: 894-904.

[25] Sanchez E, Donat E, Ribes-Koninckx C, Fernandez-Murga ML, Sanz Y. Duodenal-mucosal bacteria associated with celiac disease in children. Applied and environmental microbiology 2013; 79: 5472-5479.

[26] Lasa J, Zubiaurre I, Should Small Intestine Bacterial Overgrowth be Ruled out as a Cause of Non-Responsive Celiac Disease?: A Case Report. International Journal of Celiac Disease 2014; 2: 6769.

[27] Galatola G, Grosso M, Barlotta A, Ferraris R, Rovera L, Ariano M, et al. [Diagnosis of bacterial contamination of the small intestine using the $1 \mathrm{~g}$ [14C] xylose breath test in various gastrointestinal diseases]. Minerva gastroenterologica e dietologica 1991; 37: 16975.

[28] Tursi A, Brandimarte G, Giorgetti G. High prevalence of small intestinal bacterial overgrowth in celiac patients with persistence of gastrointestinal symptoms after gluten withdrawal. The American journal of gastroenterology 2003; 98: 839-843.

[29] Sellitto M, Bai G, Serena G, Fricke WF, Sturgeon C, Gajer P, et al. Proof of concept of microbiome-metabolome analysis and delayed gluten exposure on celiac disease autoimmunity in genetically atrisk infants. PloS one 2012; 7: e33387.

[30] Palma GD, Capilla A, Nova E, Castillejo G, Varea V, Pozo T, et al. Influence of milk-feeding type and genetic risk of developing coeliac disease on intestinal microbiota of infants: the PROFICEL study. PloS one 2012; 7: e30791. 